\title{
miR-134-5p/Foxp2/Syn1 is involved in cognitive impairment in an early vascular dementia rat model
}

\author{
XIN LIU*, RUILIN ZHANG*, ZIMEI WU, WENWEN SI, ZHENXING REN, \\ SAIXIA ZHANG, JIANHONG ZHOU and DONGFENG CHEN
}

Department of Anatomy, The Research Center of Basic Integrative Medicine, Guangzhou University of Chinese Medicine, Guangzhou, Guangdong 510006, P.R. China

Received January 16, 2019; Accepted August 7, 2019

DOI: $10.3892 /$ ijmm.2019.4331

\begin{abstract}
Forkhead box P2 (Foxp2) is a transcription factor involved in vocal learning. However, the number of previous studies that have investigated the role of Foxp2 in early vascular dementia (VD) is limited. The aim of the present study was to determine whether microRNA (miR)-134-5p/Foxp2 contributes to cognitive impairment in a chronic ischemia-induced early VD model. miR-134-5p was found to be significantly increased in the cortex in a rat VD model. Intracerebroventricular injection of miR-134-5p antagomir into VD rats prevented the loss of synaptic proteins and the development of cognitive impairment phenotypes. Histopathological analysis revealed that miR-134-5p aggravated cognitive impairment in VD rats through damage to cortical neurons and loss of synaptic proteins. Bioinformatics analysis predicted that miR-134-5p targets Foxp2 mRNA. Dual luciferase analysis and western blotting supported the prediction that miR-134-5p targets Foxp2. Furthermore, the silencing of Foxp2 significantly inhibited the effect of miR-134-5p on synaptic protein loss. Chromatin immunoprecipitation-quantitative polymerase chain reaction analysis indicated that Foxp2 binds to the synapsin I (Syn1) promoter at -400/-600 bp upstream of the transcription start site. In conclusion, the miR-134-5p/Foxp2/Syn1 axis was found to contribute to cognitive impairment in a chronic ischemia-induced early VD model, which may enable the development of new therapeutic strategies for the prevention and treatment of VD.
\end{abstract}

Correspondence to: Professor Dongfeng Chen, Department of Anatomy, The Research Center of Basic Integrative Medicine, Guangzhou University of Chinese Medicine, 232 Waihuan East Road, Guangzhou, Guangdong 510006, P.R. China

E-mail: cdf27212@21cn.com

${ }^{*}$ Contributed equally

Key words: vascular dementia, microRNA-134-5p, forkhead box P2, synapsin I, cognitive impairment

\section{Introduction}

Vascular dementia (VD) is the second most common cognitive disorder, following Alzheimer's disease (AD). VD is caused by a series of cardiac-cerebral vascular conditions that are characterized by loss of cognitive functions (1-3). Previous studies have demonstrated that the incidence of chronic cerebral hypoperfusion increases with advancing age and dementia $(4,5)$. As VD is a progressive neurodegenerative disease, treatment strategies should focus on the early stages of the pathological process. It was previously demonstrated that ischemia-induced neuronal apoptosis and synaptic reduction are major causes of early VD $(6,7)$. Accumulating evidence has shown that synaptic changes are closely associated with a number of dementia-related diseases, and are not specific to $\mathrm{AD}(8,9)$. Permanent bilateral occlusion of the common carotid artery $(2-\mathrm{VO})$ is an effective VD model in rats. A previous study reported that rats exhibited significant cognitive deficits after 4 weeks of chronic ischemia, suggesting that the early synaptic pathology of VD is associated with cognitive impairment (10). The aim of the present study was to investigate the effects of microRNA (miRNA/miR)-134-5p on cognitive deficits and elucidate the potential underlying mechanism in a chronic global cerebral ischemia rat model induced by 2-VO.

Synapses are critical for early cognition and are strongly associated with cognitive deficits, including amyloid and tau pathology $(11,12)$. Synapse-associated proteins act as synaptic morphogens, initiating functional changes; synapsin I (Syn1) and synaptosome-associated protein 25 (Snap25) are important markers involved in synaptic transmission through different mechanisms (13-15). Syn1 is implicated in the modulation of neurotransmitter release and synaptogenesis, and is involved in the transmission and regulation of synaptic information. Syn1 has a potential role in several neurological diseases, such as epilepsy and autism spectrum disorders $(16,17)$. Previous studies demonstrated that the loss of synaptic proteins is associated with VD-associated cognitive impairment $(18,19)$.

The transcription factor forkhead box P2 (Foxp2) is the only gene known to be involved in the Mendelian form of human speech and language impairment to date, and is primarily involved in influencing complex vocal cord movement and vocal learning behavior (20). Mutations in the human Foxp2 gene cause severe speech and language 
disorders $(21,22)$. In different vertebrate species, Foxp2 is highly expressed in the central nervous system during development and adulthood (23). As a transcription factor, Foxp2 regulates a number of downstream target genes and plays important roles in the development of the central nervous system, including synaptic plasticity, neurotransmission and neurite outgrowth (24). Foxp2 plays important roles in the gene networks linked to nervous system development and function. Moreover, reducing the functional dose of mouse Foxp2 led to impaired synaptic plasticity in the cortical striatum and impaired motor learning (25). Furthermore, previous studies have indicated that various genetic and environmental factors may contribute to speech and language development, and related neural developmental disorders, through the miRNA/Foxp2 regulatory network (26). However, the role of epigenetic regulation of Foxp2 in VD remains elusive.

Several miRNAs have been identified as important therapeutic targets involved in synaptic plasticity and neurodevelopment (27). The targeting of $\mathrm{Ca}^{2+1}$ calmodulin kinase II (CaMKII) by miR-219 negatively regulates arsenic-induced hippocampus structural damage and the causes impairment of memory and learning (28). However, the overexpression of miR-132 can increase the miniature excitatory postsynaptic current amplitude and dendritic protrusion width $(29,30)$. miR-12 has been reported to regulate specific synaptic plasticity during long-term olfactory habituation in Drosophila (31). miR-134 was previously reported to be widely expressed in primary cultured neurons and dendrites. miR-134 has also been reported to regulate the development of the dendritic spine by targeting Lim domain kinase 1 (LimK1), which is inhibited by brain-derived neurotrophic factor (BDNF) (32). In addition, miR-134 has been shown to exert an adverse regulatory effect on the volume of neuronal dendritic spines (33). A previous study has demonstrated that miR-134 is closely associated with the expression of synaptic proteins in the brain (34).

In the present study, 4 weeks of cerebral ischemia was induced using 2-VO in rats in order to simulate the early stage of the pathological process in VD. This model allows investigation of the role of miRNAs in cognitive impairment.

\section{Materials and methods}

Animals and VD model. In total, 18 male Sprague-Dawley rats (mean weight, 180-220 g; age, 6-7 weeks) were supplied by the Laboratory Animal Center of Guangzhou University of Chinese Medicine. The rats were housed in an environment with a constant room humidity (50-70\%), a temperature of $25 \pm 1^{\circ} \mathrm{C}$ and a 12-h light/dark cycle (lights on at 7:00 am). Water and food were available ad libitum. The experiments were approved by the Care and Use of Experimental Animals Committee of Guangzhou University of Chinese Medicine and performed according to the National Institute of Health Guide for the Care and Use of Laboratory Animals. Each cage housed 5 rats to prevent any effects of social isolation.

$V D$ surgery. The VD model was induced in rats by global cerebral ischemia using 2-VO. The rats used in the experiments were anesthetized by intraperitoneal injection $(0.15 \mathrm{ml} / 100 \mathrm{~g}$ body weight) of $100 \mathrm{mg} / \mathrm{kg}$ ketamine and $10 \mathrm{mg} / \mathrm{kg}$ xylazine. The surgery separated the bilateral common carotid arteries, which were permanently ligated with small-diameter nylon sutures. Rats in the control group underwent the same procedure, but without ligation of the common carotid arteries. The body temperature of the rats was monitored throughout the procedure and maintained at $37^{\circ} \mathrm{C}$. After surgery, the rats were placed back in their cages and normal feeding was resumed.

Intracerebroventricular (ICV) injection of the antagomir. To determine the effect of miR-134-5p on cognitive function and synaptic changes in VD rats, the ICV injection method was used to administer the miR-134-5p antagomir (5'-CCC CUCUGGUCA ACCAGUCACA-3'; Guangzhou RiboBio Co., Ltd.). The animals were randomly divided into three groups (n=6/group): The control group, the VD model group and the miR-134-5p antagomir group (2-VO + miR-134-5p antagomir). Each group of rats were continuously injected via ICV for 3 days. The miR-134-5p antagomir was dissolved in sterile double-distilled water $\left(\mathrm{ddH}_{2} \mathrm{O}\right)$ before use and a volume of $5 \mu \mathrm{l}$ was injected ( $200 \mathrm{pmol} / \mathrm{rat}$ ) into both sides of the lateral ventricle. Rats in the control and VD model groups were injected with the same volume of sterile $\mathrm{ddH}_{2} \mathrm{O}$. For the injection, the rats were anesthetized using an intraperitoneal injection $(0.15 \mathrm{ml} / 100 \mathrm{~g}$ body weight $)$ of $100 \mathrm{mg} / \mathrm{kg}$ ketamine and $10 \mathrm{mg} / \mathrm{kg}$ xylazine and placed on a stereotactic device with their backs on the panel. The antagomir was immediately injected into the lateral ventricle. According to the rat brain atlas, two small holes were drilled carefully in the skull bilaterally using a surgical drill. The stereotaxic coordinates of the ICV injection were as follows: $-0.8 \mathrm{~mm}$ anterior, $1.5 \mathrm{~mm}$ lateral and $-4.5 \mathrm{~mm}$ depth.

Morris water maze (MWM) test. To evaluate spatial memory and learning, MWM tests were conducted $24 \mathrm{~h}$ after the last ICV injection. The apparatus consisted of a black circular pool (diameter, $180 \mathrm{~cm}$ ) filled with water to a depth of $40 \mathrm{~cm}$. The maze was divided into four quadrants. The temperature of the water was $23 \pm 2^{\circ} \mathrm{C}$. A submerged circular escape platform (diameter, $20 \mathrm{~cm}$ ), $1.5 \mathrm{~cm}$ below the water surface, was placed in a random quadrant $25-30 \mathrm{~cm}$ from the edge of the pool. The platform remained in the same position throughout the experiment. On days 1-5, the rats swam freely for $90 \mathrm{sec}$ to find the platform. The rats were given 4 trials/day to train their spatial learning ability ( $\mathrm{n}=6 /$ group). If a rat failed to reach the platform, it was gently guided to the platform and placed on it for $20 \mathrm{sec}$. Each rat required a 15 -min rest before the next trial. A spatial probe test was conducted on the 6th day, during which the hidden platform was removed. Rats were allowed to swim freely for $90 \mathrm{sec}$ to determine their memory retention. After each trial, the rats were dried. The cleanliness of the water was maintained throughout the trails.

Reverse transcription-quantitative polymerase chain reaction (RT-qPCR) analysis. Rats from each group were randomly selected and anesthetized using an intraperitoneal injection $(0.15 \mathrm{ml} / 100 \mathrm{~g}$ body weight $)$ of $100 \mathrm{mg} / \mathrm{kg}$ ketamine and $10 \mathrm{mg} / \mathrm{kg}$ xylazine. The rats were then sacrificed using $\mathrm{CO}_{2}$ asphyxiation with a fill rate of $20 \%$ of the chamber volume/min with $\mathrm{CO}_{2}$. Death was confirmed using a combination of criteria, including lack of pulse, breathing, corneal reflex, response to a firm toe pinch and graying of the mucous membranes. The 
brains were quickly removed and stored at $-80^{\circ} \mathrm{C}$. Total RNA was extracted using TRIzol ${ }^{\circledR}$ (Thermo Fisher Scientific, Inc.) from cultured PC12 cells or tissue on ice for $20 \mathrm{~min}$, and then isolated using chloroform and isopropyl alcohol. The concentration and purity of the extracted RNA was determined using a UV spectrophotometer. RT was carried out using the Prime Script $^{\mathrm{TM}}$ RT reagent kit (Takara Biotechnology, Co., Ltd.), according to the manufacturer's protocol. The thermocycling conditions were $37^{\circ} \mathrm{C}$ for $15 \mathrm{~min}$ and $85^{\circ} \mathrm{C}$ for $4 \mathrm{sec}$. A specific RT primer was used for miR-134-5p (5'-GTCGTATCCAGT GCAGGGTCCGAGTATTCGCACTGGATACGA-3') and random primers were used for RT of U6. The qPCR was performed using a SYBR Premix EX TaqII kit (Takara Biotechnology, Co., Ltd.). Relative quantification of gene expression was performed using CFX96 ${ }^{\mathrm{TM}}$ Real-Time PCR Detection System (Bio-Rad Laboratories Inc.). The thermocycling conditions were as follows: $95^{\circ} \mathrm{C}$ for $30 \mathrm{sec}$, followed by 40 cycles at $95^{\circ} \mathrm{C}$ for $5 \mathrm{sec}, 55^{\circ} \mathrm{C}$ for $30 \mathrm{sec}$ and $72^{\circ} \mathrm{C}$ for $1 \mathrm{~min}$, with a final extension at $55^{\circ} \mathrm{C}$ for $5 \mathrm{sec}$. The following primers were synthesized by Sangon Biotech Co., Ltd. and used for qPCR of rno-miR-134-5p: Forward, 5'-CGCGTGTGACTG GTTGACCA-3' and reverse, 5'-AGTGCAGGGTCCGAGGT ATT-3'. The U6 qPCR primers were purchased from Sangon Biotech Co., Ltd. (cat. no. MQP-0101). Differences in gene expression were analyzed using the $2^{-\Delta \Delta \mathrm{Cq}}$ method (35). U6 was used as an internal control for the detection of miRNA. Each experiment was repeated three times.

Bioinformatics prediction of the target genes of miR-134-5p. The target genes of Rattus norvegicus (rno)-miR-134-5p were predicted using bioinformatics tools, including TargetScan (version 7.2; www.targetscan.org/mmu_71), miRBase (version 22.1; www.mirbase.org) and miRWalk (version Nov/2018 Release; http://mirwalk.umm.uni-heidelberg.de). The predicted gene targets were screened using Venn diagrams and the DAVID Bioinformatics Resources 6.8 (david.ncifcrf.gov) for gene function analysis. The target genes of rno-miR-134-5p were verified using western blotting and dual-luciferase reporter gene analysis.

Histopathological observation using hematoxylin and eosin $(H \& E)$ staining. After 4 weeks of $2-\mathrm{VO}$ and following the MWM test, rats in each group were randomly selected and anesthetized. Rats were immediately perfused transcardially with cold normal saline followed by $\sim 100 \mathrm{ml}$ of cold $4 \%$ paraformaldehyde to prefix the brain tissue. The brains were washed with cold saline and fixed in $4 \%$ paraformaldehyde at $4^{\circ} \mathrm{C}$ overnight. Paraffin sections of $4 \mu \mathrm{m}$ were used for H\&E, TUNEL and immunohistochemical staining. Sections from the cortex and hippocampus were stained using an H\&E staining kit (Beijing Solarbio Science \& Technology). The sections were visualized using a light microscope (Leica dMI400; Leica Microsystems GmbH) and photographed. Each experiment was repeated three times.

TUNEL staining. TUNEL staining was used to identify apoptotic cells in the cortex, following the manufacturer's protocol (one-step TUNEL apoptosis assay kit; Beyotime Institute of Biotechnology). Nuclei were stained with DAPI (1:100; Beijing Solarbio Science \& Technology) for $10 \mathrm{~min}$ at $37^{\circ} \mathrm{C}$ in a humidified atmosphere in the dark. Images of apoptotic cells were captured using a confocal laser scanning microscope (LSM 800; Carl Zeiss AG) at x200 magnification. Each experiment was repeated three times.

Immunohistochemical staining. Immunohistochemical staining was performed using a Histostain ${ }^{\mathrm{TM}}$-Plus kit (Beijing Biosynthesis Biotech Co., Ltd.) according to the manufacturer's instructions. The dehydrated and transparent tissue block was placed in dissolved paraffin, and after the tissue block was completely immersed in paraffin, it was embedded and sliced. Paraffin sections were incubated with anti-Syn1 primary antibody (1:100; cat. no. ab8; Abcam) at $4^{\circ} \mathrm{C}$ overnight, and incubated with a biotinylated secondary antibody for $20 \mathrm{~min}$ at $37^{\circ} \mathrm{C}$. The staining was examined and images were captured using a light microscope (Leica dMI400; Leica Microsystems $\mathrm{GmbH})$ at $\mathrm{x} 400$ magnification. In total, five fields of view were randomly selected on each section to measure the expression level of Syn1. Each experiment was repeated three times.

Western blot analysis. Proteins were extracted using RIPA lysis buffer (Thermo Fisher Scientific, Inc.) from cultured PC12 cells or tissue (pulverized frozen fresh cortex) on ice for $20 \mathrm{~min}$. The supernatants were obtained by centrifugation at $12,000 \mathrm{x} \mathrm{g}$ at $4^{\circ} \mathrm{C}$ for $20 \mathrm{~min}$. Protein concentrations were quantified using a bicinchoninic acid protein assay (Fdbio Science). Equal amounts of protein (20-50 $\mu \mathrm{g} /$ lane) were separated using $10 \%$ SDS-PAGE gels and transferred onto nitrocellulose membranes after electrophoresis. After blocking with 5\% skimmed milk in TBS containing $0.01 \%$ Tween-20 (TBST) at room temperature for $2 \mathrm{~h}$, the blots were incubated with anti-Foxp2 (1:1,000; cat. no. ab16046; Abcam), anti-Snap25 (1:1,000; cat no. A0986; ABclonal Biotech Co., Ltd.), anti-Syn1 (1:1,000; cat. no. ab8; Abcam) and anti- $\beta$-actin (1:5,000; cat. no. ab8227; Abcam) primary antibodies at $4^{\circ} \mathrm{C}$ overnight. Following washing with TBST, the blots were incubated with a secondary horseradish peroxidase-conjugated goat anti-rabbit antibody $(1: 1,000$; cat. no HAF008, R\&D Systems, Inc.) for $1 \mathrm{~h}$ at room temperature and washed with TBST. Protein bands were visualized using an enhanced chemiluminescence kit (Beyotime Institute of Biotechnology), according to the manufacturer's protocol. Protein bands were analyzed using ImageJ software (version 1.4; National Institutes of Health). The expression of the target protein was calculated by comparing the gray value of each group with the corresponding internal reference gene. Each experiment was repeated three times.

PC12 cell culture. PC12 rat pheochromocytoma cells are frequently used as a model in neuronal research (36). PC12 cells were purchased from Procell Life Science \& Technology Co., Ltd. and were cultured at $37^{\circ} \mathrm{C}$ in a $5 \% \mathrm{CO}_{2}$ incubator in RPMI 1640 medium (Gibco; Thermo Fisher Scientific, Inc.), 10\% FBS (Gibco; Thermo Fisher Scientific, Inc.) and $1 \%$ solution penicillin/streptomycin (Thermo Fisher Scientific, Inc.). The medium was replaced every 3 days. PC12 cells were plated into 6- or 24-well plates at a density of $9 \times 10^{5}$ or $1.5 \times 10^{5}$ cells/well, respectively.

Luciferase reporter assay. PC12 cells were seeded in 24-well plates at a density of $1 \times 10^{5}$ cells/well and were subsequently 
transfected with miR-134-5p mimic, miR-134-5p inhibitor, miR-134-5p mimic-NC or miR-134-5p inhibitor-NC (Guangzhou RiboBio Co., Ltd.), and co-transfected with pLUC-Foxp2-wild-type (WT) 3'-untranslated region (UTR) or pLUC-Foxp2-mutant (MUT) 3'-UTR plasmids (Shenzhen Huaan Ping Kang Bio Technology Co., Inc.) using Lipofectamine ${ }^{\circledR} 2000$ (Thermo Fisher Scientific, Inc.), according to the manufacturer's protocol. In the pLUC-Foxp2 cloning vector, miRNA target sites, predicted using TargetScan software (version 7.2; http://www.targetscan.Org/vert_71/), were inserted after the Renilla luciferase region. The final concentration of miR-134-5p mimic and miRNA mimic negative control transfected into PC12 cells was $50 \mathrm{nM}$, and the final concentration of miR-134-5p inhibitor and miRNA inhibitor negative control was $100 \mathrm{nM} ; 200 \mathrm{ng}$ plasmid was used for transfection. PC12 cells were lysed and the luciferase activity was quantified using the Dual-Luciferase ${ }^{\circledR}$ Reporter assay kit (Promega Corporation), according to the manufacturer's protocol, $48 \mathrm{~h}$ after transfection. Each experiment was repeated three times.

Transfection of PC12 cells with miR-134-5p mimic, miR-134-5p inhibitor and the respective miRNA negative controls (miRNA mimic-NC and miRNA inhibitor-NC) or siRNA (siR)-Foxp2. PC12 cells were seeded into 6-well plates at a density of $9 \times 10^{5}$ cells/well 1 day before transfection in order to reach a confluence of $60-80 \%$. Transfection of miR-134-5p mimic (5'-UGUGACUGGUUGACCAGAGGGG-3' and 5'-ACACUG ACCAACUGGUCUCCCC-3'), miR-134-5p mimic-NC (cat. No miR1N0000001-1-5; sequence unavailable; Guangzhou RiboBio Co., Ltd.), miR-134-5p inhibitor (5'-CCCCUCUGG UCAACCAGUCACA-3'), miR-134-5p inhibitor-NC (cat. no miR2N0000001-1-5; sequence unavailable; Guangzhou RiboBio Co., Ltd.) or siRNA-Foxp2 (siR-Foxp2-001, AGC AGCAACAACTACAAGA; siR-Foxp2-002, CAAAGCTTC ACCGCCAATA; siR-Foxp2-003, CGACATTCAGACAAA TACA) was performed using Lipofectamine ${ }^{\circledR} 2000$ (Thermo Fisher Scientific, Inc.), according to the manufacturer's protocol. The final concentration of mimic, mimic-NC and siRNAs was $50 \mathrm{nM}$, and the final concentration of inhibitor and inhibitor-NC was $100 \mathrm{nM}$. Cells were lysed with RIPA buffer $48 \mathrm{~h}$ after transfection for western blot analysis. Each experiment was repeated three times.

Chromatin immunoprecipitation (ChIP)-qPCR. Using ChIP assays, the molecular interaction between Foxp2 and the Syn1 promoter binding site was investigated. Nerve growth factor (NGF; $50 \mathrm{ng} / \mathrm{ml}$ ) was used to induce PC12 cells for 1 week. According to the manufacturer's instructions (Magna ChIP ${ }^{\mathrm{TM}}$ A; EMD Millipore), the culture medium was removed and $1 \%$ formaldehyde was added for crosslinking for $10 \mathrm{~min}$ at $37^{\circ} \mathrm{C}$; $2 \mathrm{ml}$ of $0.125 \mathrm{M}$ glycine was added to quench unreacted formaldehyde. The cells were washed using ice-cold PBS twice and then collected and lysed in SDS Lysis Buffer (EMD Millipore). Chromatin was extracted from the nuclei. The chromatin was then sheared to $200-1,000 \mathrm{bp}$ fragments by ultrasonication at $4^{\circ} \mathrm{C}$ for $40 \mathrm{~min}$, and the effect of ultrasonic crushing was examined by agarose gel electrophoresis. The Foxp2 antibody (Abcam) was applied to immunoprecipitated DNA by using magnetic protein $\mathrm{G}$ beads. Following reverse crosslinking, ChIP-DNA complexes were extracted, washed and eluted using $100 \mu \mathrm{l} \mathrm{ChIP}$ Elution Buffer (EMD Millipore) with $1 \mu 1$ protease K (EMD Millipore). DNA was purified by Spin Filter. The ChIP-DNA fragments were directly used in qPCR assays with specific primers for the promoter region of the Syn1. GAPDH was used as an internal control for the detection of mRNA. The primers used were as follows: Syn1-1: Forward, 5'-GGACCCCTA AGTTCCTTCCTCCA-3' and reverse, 5'-AGACACAAACAT TGGCAAAGGTGG-3'; Syn1-2: Forward, 5'-CTCCCAAAT CCGCATGGGGT-3' and reverse, 5'-GTCTCCTCTTGGCTT TGGGGATAGT-3'; Syn1-3: Forward, 5'-CTGAGGCAGTAT CAGGGCACAG-3' and reverse, 5'-CTGCCTTCTCAGCGC AGCC-3'; and GAPDH: Forward, 5'-GTCCATGCCATCACT GCCACTC-3' and reverse, 5'-CGCCTGCTTCACCACCTT CTTG-3'. ChIP was analyzed by qPCR using the CFX96 ${ }^{\mathrm{TM}}$ Real-Time PCR Detection System (Bio-Rad Laboratories, Inc.). Each experiment was repeated three times.

Statistical analysis. All data are presented as the mean \pm standard deviation. Data were analyzed using Excel 2017 (Microsoft Corporation) and GraphPad Prism7 (version 7.0; GraphPad Software, Inc.). Comparisons between two groups were performed using a t-test. Comparison among multiple groups were analyzed using one-way ANOVA followed by Tukey's multiple comparisons test for post hoc analysis. $\mathrm{P}<0.05$ was considered to indicate a statistically significant difference.

\section{Results}

miR-134-5p inhibitor relieves cognitive dysfunction in VD rats following 4 weeks of chronic ischemia. A rat model of dementia was produced using 2-VO for 4 weeks. In order to verify whether miR-134-5p could affect the spatial learning and memory ability of VD rats, MWM experiments were conducted after 3 days of lateral ventricular injection of the miR-134-5p antagomir (n=6/group). During MWM training, the escape latency of rats decreased significantly, indicating that the platform could be found with training. In consecutive trials, the VD rats were impaired while training to find the submerged escape platform, as indicated by longer escape latencies compared with the control group. The escape latency of the miR-134-5p antagomir group was shorter compared with that of the VD model group; this was also the case on the 5th day (Fig. 1A-C). In the MWM test, the number of rats crossing over the platform position, and the percentage of time in the target quadrant relative to the total escape latency time in the pool, was significantly decreased in VD model rats and significantly increased in the miR-134-5p antagomir group compared with the control group (Fig. 1D and E). Representative movement traces of the MWM test on the 6th day revealed that the number of VD model rats crossing the platform was significantly lower compared with that of rats in the control group. In addition, the number of miR-134-5p antagomir rats crossing the platform was significantly higher compared with in the VD model group (Fig. 1F). Using the MWM experiment, it was demonstrated that rats exhibited spatial learning impairment following 4 weeks of chronic ischemia. Behavioral tests also indicated that inhibiting miR-134-5p could relieve cognitive dysfunction, as well as enhance learning and memory ability, in VD rats. 
A

$\left.\prod_{\text {D0 }}\right|_{\text {D7 }} \begin{gathered}\text { chronic ischemia } \\ \text { cheeks }\end{gathered}$

B

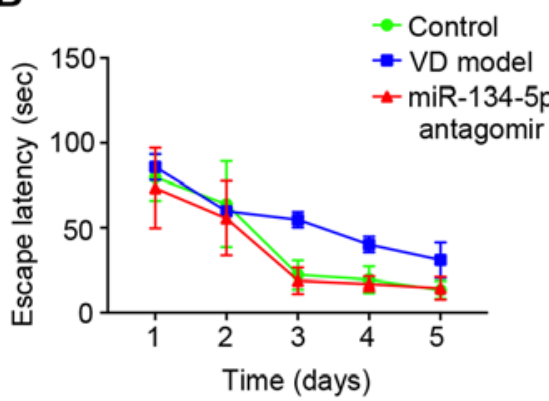

E

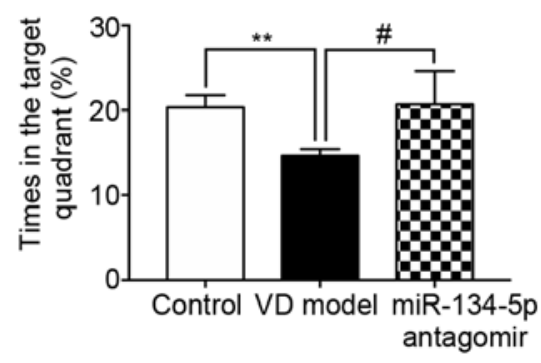

C

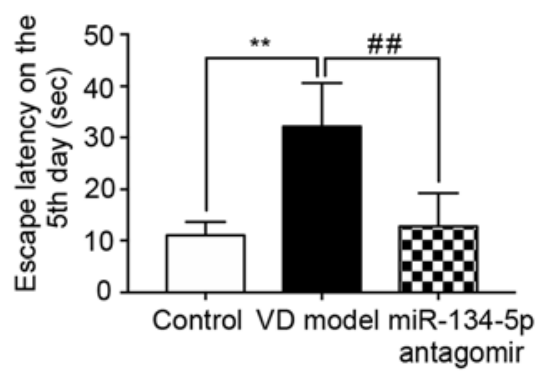

F

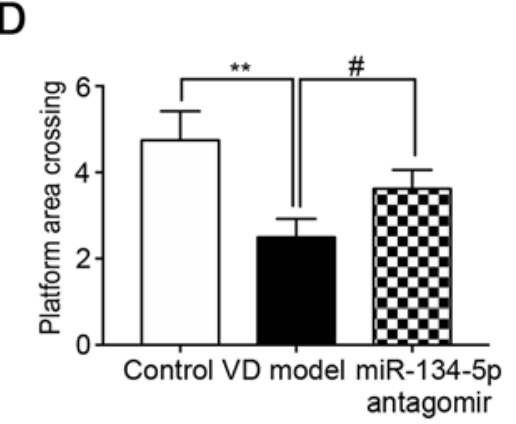

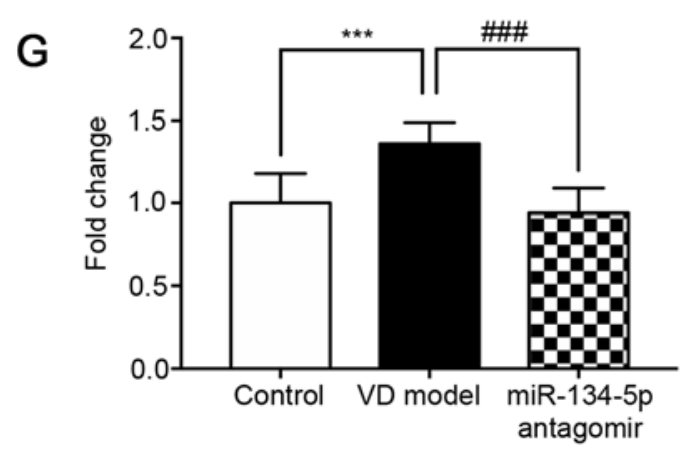
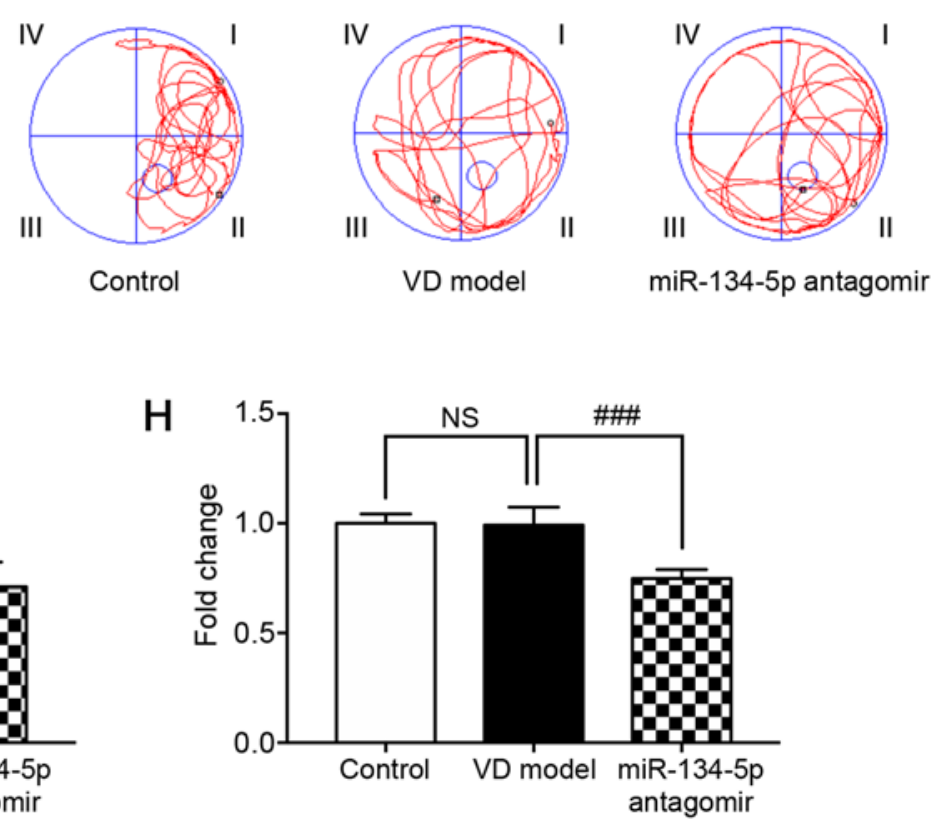

Figure 1. Lateral ventricle injection of miR-134-5p antagomir attenuates learning and memory deficits in rats following 4 weeks of chronic ischemia. (A) Timeline of the experiment ( $\mathrm{n}=6 /$ group). (B) Escape latency was assessed using the MWM test over 5 days. (C) Escape latency of the control group, the VD model group and the miR-134-5p antagomir group on the 5th day. (D) Number of platform crossings measured within 90 sec. (E) Percentage of time in the target quadrant relative to the total escape latency time in the pool during the MWM test on the 6th day. (F) Representative movement traces of the MWM test on the 6th day. ( $\mathrm{G}$ and $\mathrm{H}$ ) miR-134-5p expression was quantified using reverse transcription-quantitative polymerase chain reaction analysis (n=3/group). The expression of miR-134-5p was found to be significantly increased in the cortical tissue of VD model rats compared with the control group. No significant differences were found in the hippocampus. Data were analyzed using one-way ANOVA and are presented as the mean \pm standard deviation. ${ }^{* *} \mathrm{P}<0.01$, ${ }^{* * * *} \mathrm{P}<0.001$ vs. the control group; ${ }^{\#} \mathrm{P}<0.05,{ }^{\# \#} \mathrm{P}<0.01,{ }^{\# \#} \mathrm{P}<0.001$ vs. the VD model group. MWM, Morris water maze; VD, vascular dementia; miR, microRNA.

miR-134-5p expression is increased in the cortex of VD rats. To determine whether miR-134-5p is upregulated in the brains of VD rats, RT-qPCR analysis was performed ( $\mathrm{n}=3$ /group). The results demonstrated that the expression of miR-134-5p in the cortical tissue of VD model rats was increased significantly compared with the control group (Fig. 1G). No significant differences were found in the hippocampus of VD model rats compared with the control group (Fig. 1H). These results revealed that miR-134-5p is implicated in chronic ischemia-induced VD. These results also indicated that miR-134-5p is differentially expressed in the cortex, but not in the hippocampus, of VD rats.

Histopathological changes and apoptosis levels are increased in the cortex of VD rats. Histopathological changes in the cortex and the cornu ammonis 1 (CA1) region of the hippocampus were observed following staining with $\mathrm{H} \& \mathrm{E}$ at 4 weeks after 2-VO, and are shown in Fig. 2 ( $n=3 /$ group). In the cortex of VD rats, neuronalcell loss, shrinkage, dark staining of neurons and marked vacuolar changes were observed in the cortex. Additionally, the space between neurons was enlarged. The nucleus was pyknotic and the structure was not clear. Inhibition of miR-134-5p attenuated chronic hypoperfusion-induced neuronal cell injury. The structure of the nucleus in the hippocampal CA1 region of VD rats was not sufficiently clear compared with the control group; however, no significant damage in hippocampal CA1 neurons was observed in VD rats. Neurons undergoing apoptosis were detected using TUNEL staining ( $n=3$ /group). As shown in Fig. 3A and B, there were more TUNEL-positive neurons in 

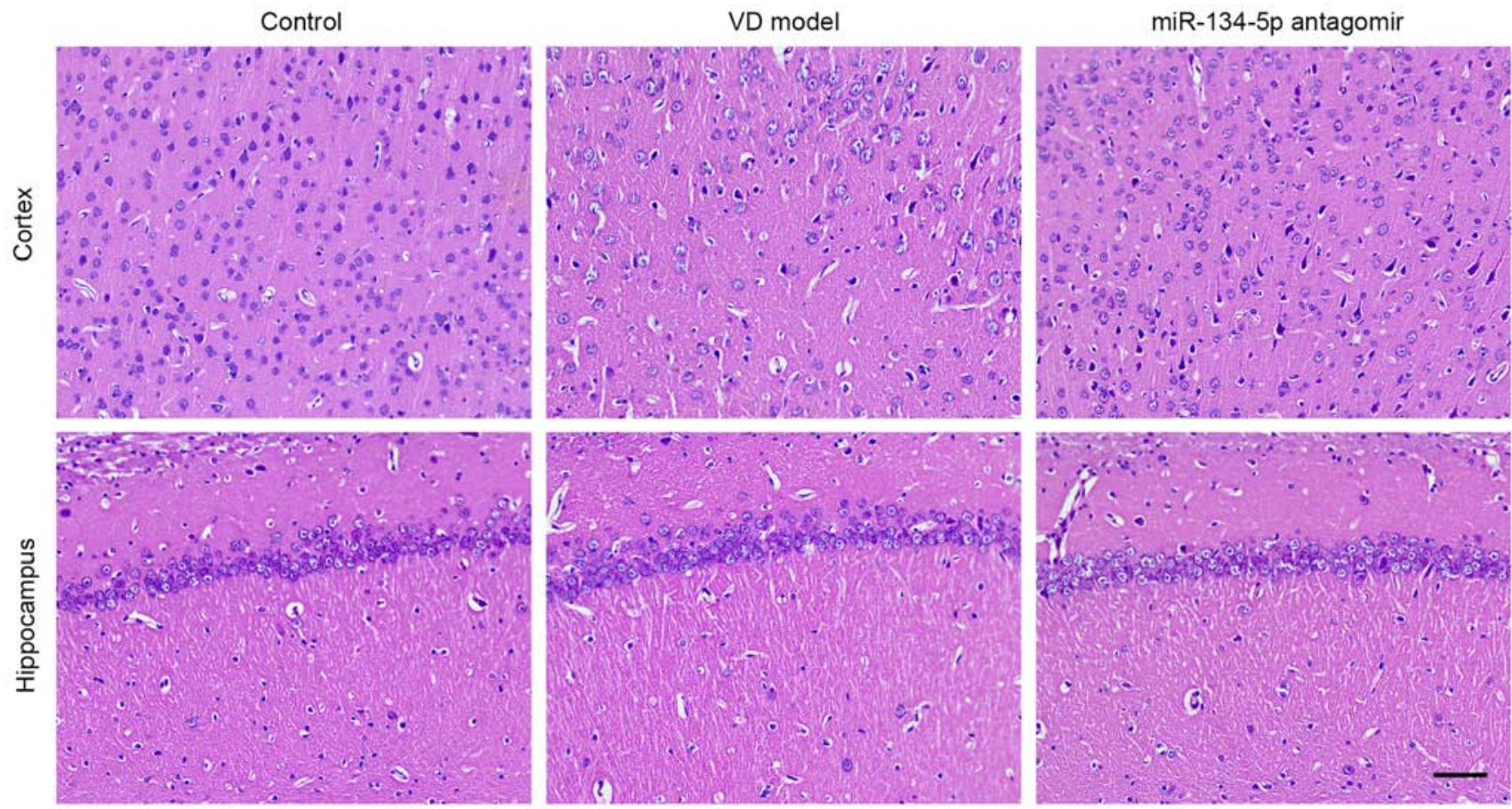

Figure 2. Histopathological changes 4 weeks after permanent bilateral ligation of the common carotid arteries (hematoxylin and eosin staining; magnification $\mathrm{x} 200$ ). Representative images showing changes in the morphology of the cortex and the CA1 region of the hippocampus in the control group, the VD model group and the miR-134-5p antagomir group (n=3/group). Scale bar, $50 \mu \mathrm{m}$. VD, vascular dementia; miR, microRNA; CA1, cornu ammonis 1 .

the cortex of VD rats compared with the hippocampal CA1 region. By contrast to VD rats, the miR-134-5p antagomir group had markedly fewer TUNEL-positive neurons. These results suggested that cortical injury was more prominent compared with that of the hippocampus in early VD rats following chronic ischemia for 4 weeks.

Foxp2 is a potential target of miR-134-5p. Bioinformatics analysis predicted that the miR-134-5p sequence GUCAGUG was associated with the Foxp2 gene sequence CAGUCAC (Fig. 4A). To assess the direct effect of miR-134-5p on Foxp2 gene expression, dual-luciferase reporters carrying the miR-134-5p target site in pLUC-Foxp2 were constructed (Fig. 4B). This analysis demonstrated that an miR-134-5p mimic significantly decreased the luciferase activity of Foxp2. By contrast, the miR-134-5p mimic did not reduce luciferase activity when the miR-134-5p seed sequence at the 3'UTR of Foxp2 was mutated (Fig. 4C). To determine whether the miRNA mimic and inhibitor transfections were successful, RT-qPCR analysis was performed ( $n=3 /$ group). The results revealed that the expression of miR-134-5p was significantly increased in the mimic group compared with that in the mimic-NC group, and significantly reduced in the inhibitor group compared with that in the inhibitor-NC group (Fig. 4D). Western blot analysis identified Foxp2 as a potential target of miR-134-5p. The expression of Foxp2 was reduced in the mimic group compared with the mimic-NC group and increased in the inhibitor group compared with the inhibitor-NC group (Fig. 4E). These results indicated that miR-134-5p regulates Foxp2 directly.

miR-134-5p inhibitor relieves synaptic reduction in vivo and in vitro. Western blotting verified that the expression levels of the representative synaptic-associated proteins, Syn1 and
Snap25, were significantly reduced in the cortex of VD model rats compared with the control group, and were markedly increased in the cortex of miR-134-5p antagomir rats compared with VD model rats (Fig. 5A). According to the results of immunohistochemical staining ( $\mathrm{n}=3 /$ group), the expression of Syn1 was significantly reduced in the cortex of VD rats compared with the control group. The miR-134-5p antagomir significantly enhanced Syn1 expression compared with the VD model group (Fig. 5B). To determine whether Foxp2 expression in the cortex was associated with synaptic reduction in the development of early VD and the targeting of miR-134-5p to Foxp2 in vivo, western blot analysis was performed to detect Foxp2 expression ( $n=3$ /group). The results indicated that the expression of Foxp2 in the cortex of VD rats was significantly decreased compared with that in the control group, and was significantly higher in the cortex of miR-134-5p antagomir rats compared with VD model rats (Fig. 5C). In order to further elucidate the effect of miR-134-5p on loss of synaptic proteins, miR-134-5p was transfected into PC12 cells. The results of the RT-qPCR analysis demonstrated that the miRNA mimic and inhibitor transfections were successful ( $n=3 /$ group). The expression of miR-134-5p was significantly increased in the mimic group compared with the mimic-NC group, and significantly reduced in the inhibitor group compared with the inhibitor-NC group (Fig. 5D). Western blotting revealed that the expression levels of Snap25 and Syn1 were decreased in the miR-134-5p mimic group and increased in the miR-134-5p inhibitor group, compared with the respective miRNA NC groups (Fig. 5E). These results indicated concomitant synaptic protein loss in VD rats, and the downregulation of miR-134-5p relieved synaptic protein loss in the cortex of VD rats. In addition, these results indicated that miR-134-5p regulated the loss of synaptic proteins in vivo and in vitro, and the targeting of miR-134-5p to Foxp2 was further verified in vivo. 
A
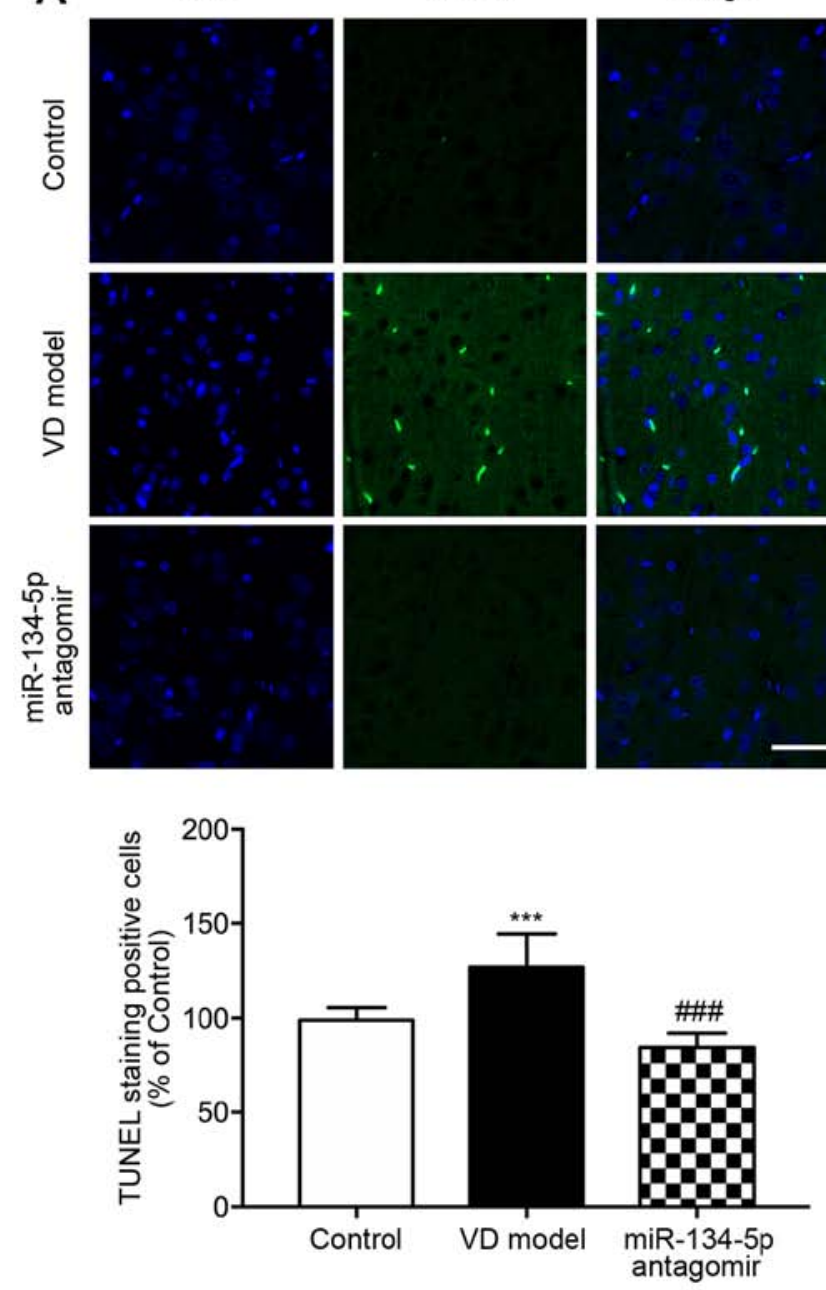

B
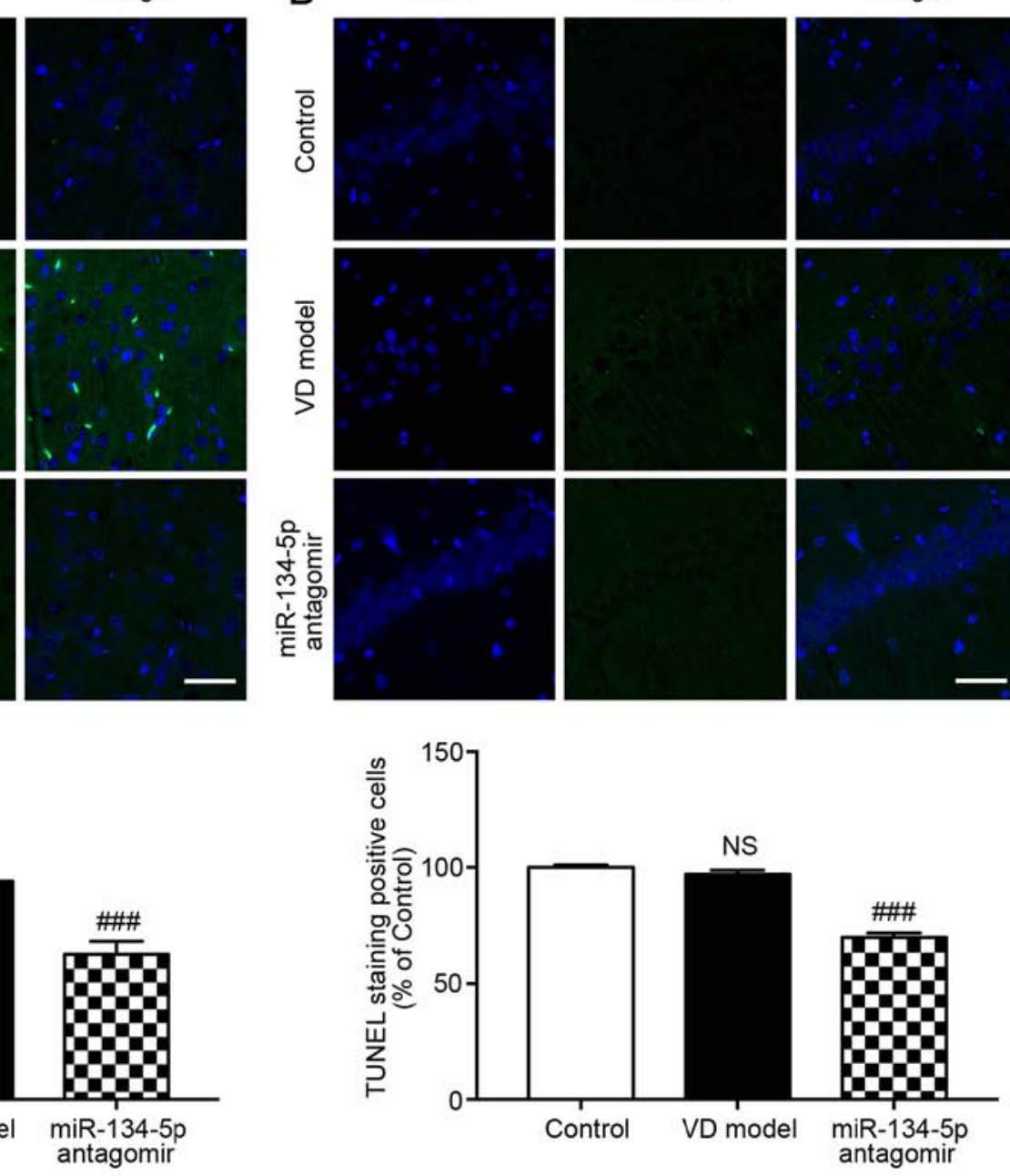

Figure 3. TUNEL staining was used to determine the extent of cell apoptosis. Expression of apoptotic factors (A) in the cortex of VD rats and (B) in the CA1 region of the hippocampus. Data were analyzed using one-way ANOVA and are presented as the mean \pm standard deviation ( $\mathrm{n}=3 / \mathrm{group}$ ). Scale bar, $50 \mu \mathrm{m}$. ${ }^{* * *} \mathrm{P}<0.001$ vs. the control group; ${ }^{\# \# \#} \mathrm{P}<0.001$ vs. the VD model group. VD, vascular dementia; miR, microRNA; CA1, cornu ammonis 1 .

Silencing Foxp2 decreases the expression of synaptic-associated proteins. To investigate whether miR-134-5p regulates the expression of synaptic-associated proteins by targeting Foxp2, siR-Foxp2-001, siR-Foxp2-002 or siR-Foxp2-003 were transfected into PC12 cells to determine the effect of Foxp2 on the expression of Syn1 and Snap25. Western blot analysis revealed that siR-Foxp2-002 significantly suppressed the expression of Foxp2 at the protein level compared with siR-NC (Fig. 6A). The expression of synaptic-associated proteins, such as Syn1 and Snap25, was decreased in the siR-Foxp2-002 group compared with the siR-NC group (Fig. 6B). These data confirmed that the downregulation of Foxp2 significantly inhibited the expression of the synapse-associated proteins Syn1 and Snap25, thereby affecting the morphology and function of synapses. Due to the direct targeting effect of miR-134-5p on Foxp2, these results also indicated that miR-134-5p affects the expression of synaptic-associated proteins by regulating Foxp2, rather than by directly regulating synaptic-associated proteins.

Syn1 is a potential downstream target of Foxp2. To investigate whether Syn1 is a downstream target of Foxp2, ChIP assays were performed. The model group was PC12 cells induced with NGF $(50 \mathrm{ng} / \mathrm{ml})$ for 1 week. The control group received no treatment. Verified using an RT-qPCR assay, the level of DNA fragment in the model group was significantly increased compared with the control group (Fig. 6C). ChIP analysis revealed the binding of Foxp2 to the Syn1 promoter at $-400 /-600$ bp upstream of the transcription start site in PC12 cells induced by NGF. These results indicated that Foxp2 regulates synaptic changes through its interaction with the Syn1 promoter.

\section{Discussion}

VD is often accompanied by changes in cognitive function, which is closely associated with early synaptic changes (37). Ischemic VD leads to cognitive dysfunction $(38,39)$. However, it is not known how miR-134-5p regulates cognitive impairment in VD caused by chronic ischemia. In the present study, 2-VO was used to create a VD model and was found to mimic several prominent pathological characteristics of early VD. Whether miR-134-5p/Foxp2/Syn1 participates in cognitive dysfunction in the early stage of VD was also investigated. The principal findings of the present study were as follows: i) miR-134-5p was increased in the cortex of VD rats; ii) downregulation of miR-134-5p significantly relieved 
A
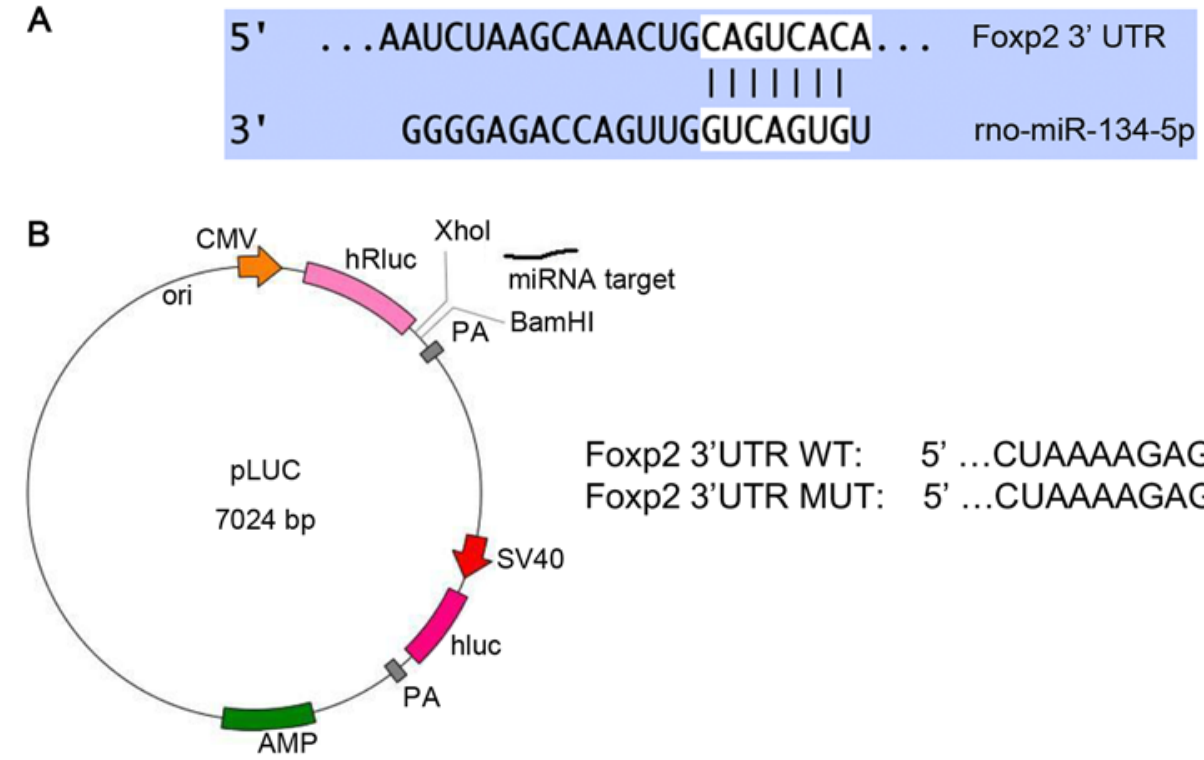

Foxp2 3'UTR WT: $\quad$ ', ...CUAAAAGAGUUCACUCAGUCACA... Foxp2 3'UTR MUT: 5 ' ...CUAAAAGAGUUCACUGUCAGUGA...

C
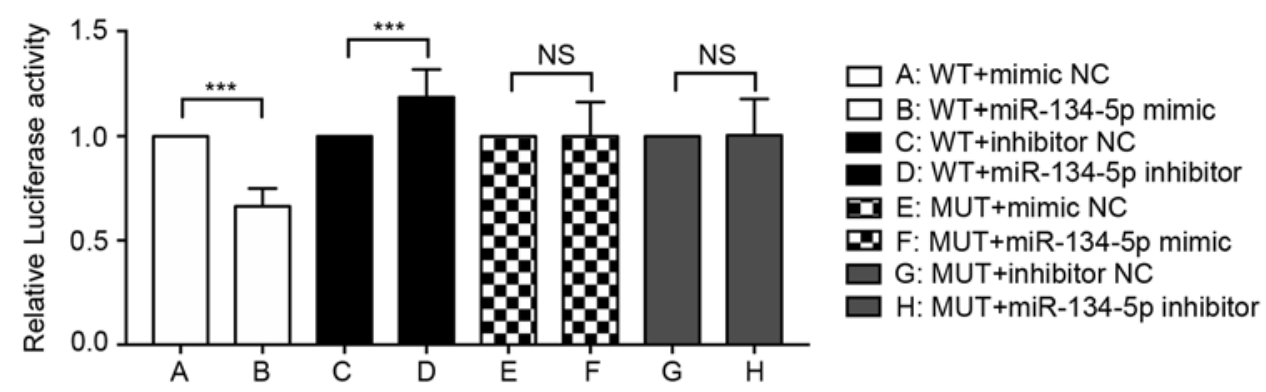

D

E
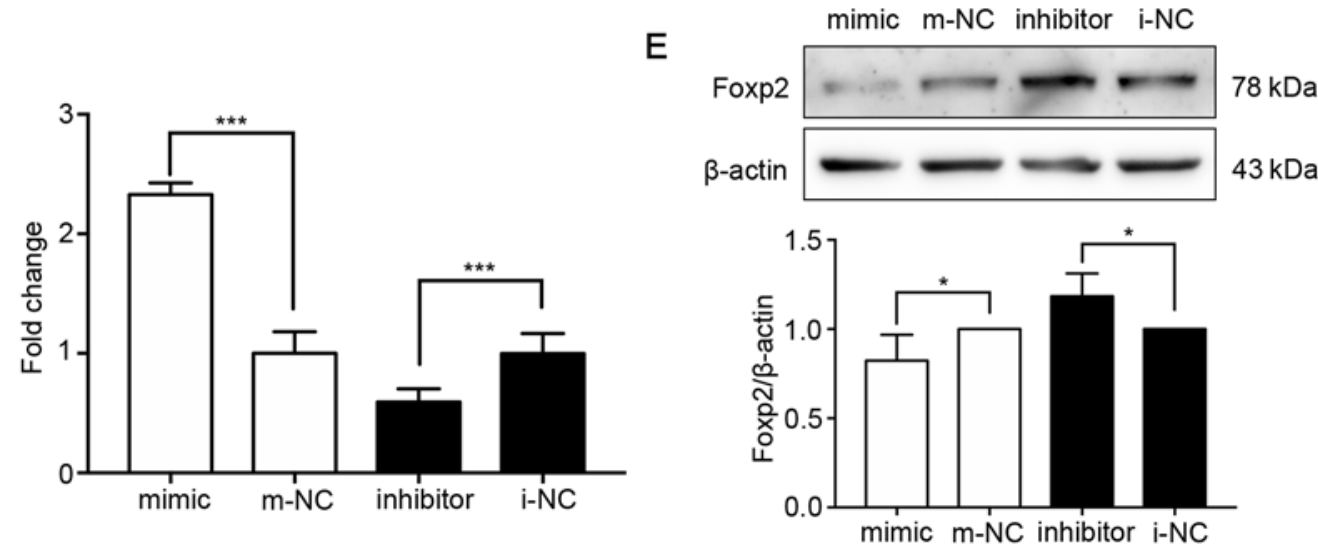

Figure 4. Foxp2 is a potential target of miR-134-5p. (A) miR-134 binding sites in the 3'-UTR of Foxp2. The target sites were analyzed by Targetscan, miRBase and miRWalk. (B) Construction of pLUC-Foxp2-WT and pLUC-Foxp2-MUT containing the miR-134-5p target sites in the Foxp2 3'-UTR. (C) pLUC-Foxp2-WT and the corresponding mutant construct pLUC-Foxp2-MUT were co-transfected into PC12 cells together with miR-134-5p mimic, inhibitor or the corresponding miRNA negative controls. The relative luciferase activity of PC12 cells indicated that there was an association between miR-134-5p and Foxp2. (D) RT-qPCR analysis demonstrated the miRNA mimic and inhibitor transfections were effective and successful (n=3/group). (E) Western blot analysis revealed a decrease in the expression of Foxp2 in PC12 cells transfected with miR-134-5p mimic and an increase in the expression of Foxp2 in cells transfected with the miR-134-5p inhibitor. Data were analyzed using one-way ANOVA and are presented as the mean \pm standard deviation $(\mathrm{n}=3 / \mathrm{group}){ }^{*} \mathrm{P}<0.05,{ }^{* * * *} \mathrm{P}<0.001$ vs. the respective miRNA NC groups. Foxp2, forkhead box P2; miR, microRNA; m-NC, miRNA mimic negative control; i-NC, miRNA inhibitor negative control; WT, wild-type; MUT, mutant; UTR, untranslated region.

the cognitive deficit of VD rats; iii) Foxp2 was identified as a direct target of miR-134-5p; and iv) Foxp2 acts as a transcription factor and Syn1 may be a downstream target of Foxp2. miR-134-5p was found to indirectly regulate the loss of synaptic proteins through the direct targeting of Foxp2. These results suggested that the miR-134-5p/Foxp2/Syn1 signaling pathway may play an important role in cognitive dysfunction in early VD.
2-VO is a well-characterized model for investigating the cognitive and histopathological consequences of chronic cerebral hypoperfusion (4). This model more accurately simulates VD caused by vascular factors, which is similar to the pathological process in the brain tissue in chronic cerebral hypoperfusion (40). In the present study, rats were found to exhibit spatial learning impairment and synaptic protein loss following 4 weeks of chronic ischemia. Several miRNAs may 
A

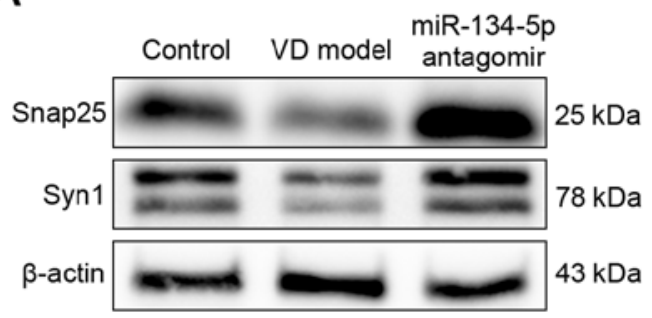

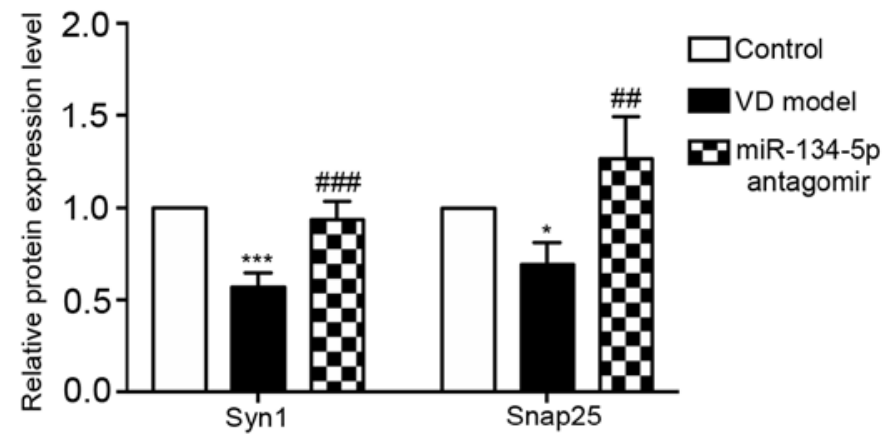

B

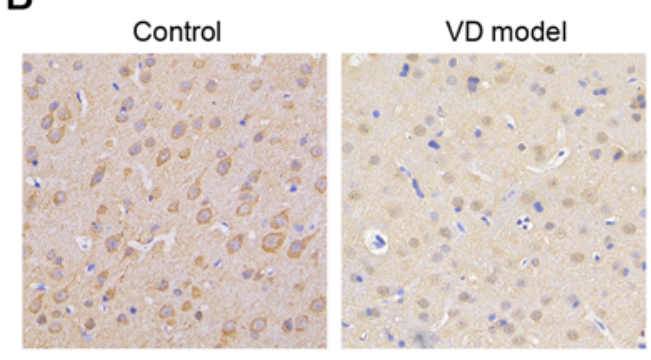

miR-134-5p antagomir

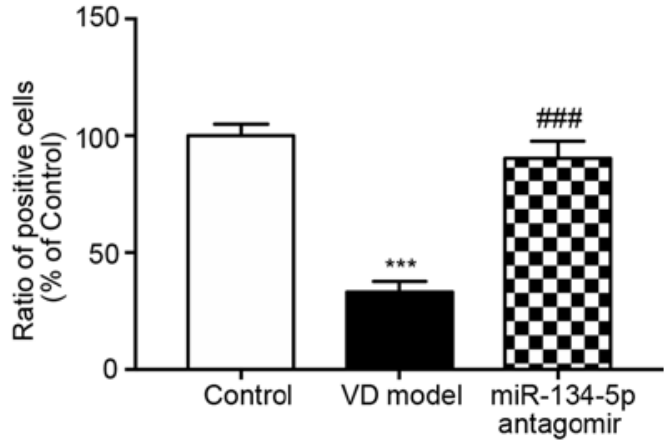

C
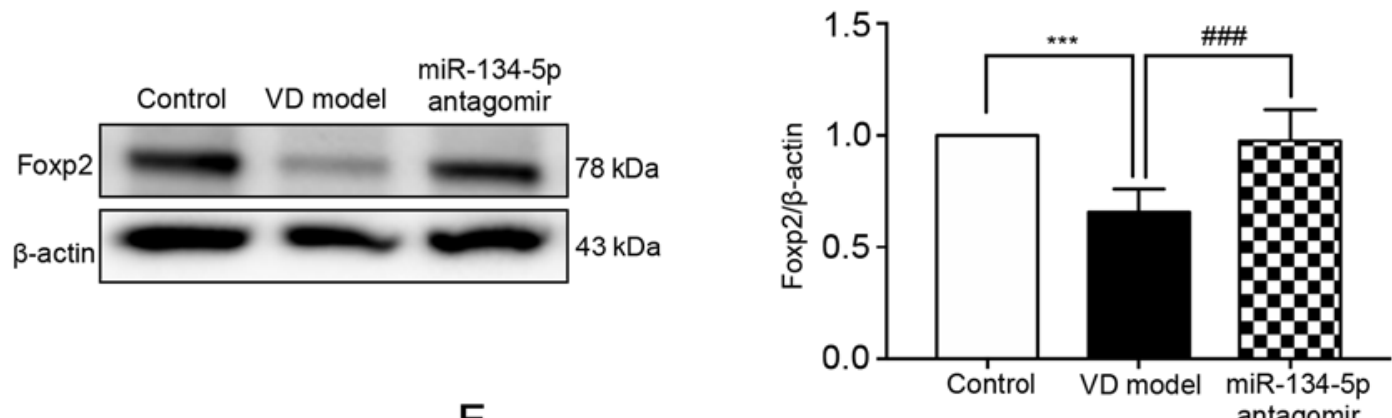

E
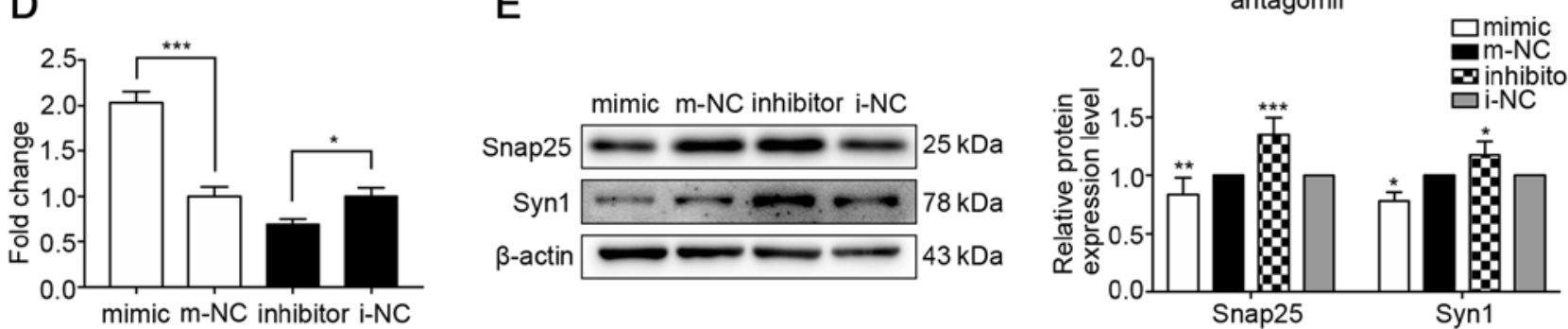

Figure 5. Inhibiting the expression of miR-134-5p reduces the loss of synaptic proteins in vivo and in vitro. Expression of (A) Syn1 and Snap25. Two bands for Syn1 are shown, as the Syn1 gene is subject to alternative splicing, producing two isoforms (synapsin Ia and Ib) which are differentially expressed in animal tissues and cells. ${ }^{*} \mathrm{P}<0.05,{ }^{* * * *} \mathrm{P}<0.001$ vs. the control group; ${ }^{\# /} \mathrm{P}<0.01,{ }^{\# \# \#} \mathrm{P}<0.001$ vs. the VD model group. (B) miR-134-5p antagomir attenuated the loss of the Syn1 protein in the cortex of VD rats. Immunohistochemical staining of paraffin sections was used to observe the expression of Syn1 (n=3/group). ${ }^{* * * *} \mathrm{P}<0.001$ vs. the control group; ${ }^{\# \# \#} \mathrm{P}<0.001$ vs. the VD model group. (C) Foxp2 was determined using western blotting ( $\left.\mathrm{n}=3 / \mathrm{group}\right) .{ }^{* * * *} \mathrm{P}<0.001$ vs. the control group; ${ }^{\# \# \# ~} \mathrm{P}<0.001$ vs. the VD model group. (D) RT-qPCR analysis confirmed that the miRNA mimic and inhibitor transfections were effective and successful ( $\mathrm{n}=3$ /group). ${ }^{*} \mathrm{P}<0.05,{ }^{* * * *} \mathrm{P}<0.001$ vs. the respective miRNA NC groups. (E) Western blot analysis revealed decreased expression of Syn1 and Snap25 in PC12 cells transfected with miR-134-5p mimic and an increased expression of Syn1 and Snap25 in cells transfected with miR-134-5p inhibitor (n=3/group). "P<0.05, ${ }^{* *} \mathrm{P}<0.01,{ }^{* * *} \mathrm{P}<0.001$ vs. the respective miRNA NC groups. Data were analyzed by one-way ANOVA and are presented as the mean \pm standard deviation. Scale bar, $50 \mu \mathrm{m}$. VD, vascular dementia; miR, microRNA; m-NC, miRNA mimic negative control; i-NC, miRNA inhibitor negative control; Syn1, synapsin I; Snap25, synaptosome-associated protein 25; Foxp2, forkhead box P2.

be used as diagnostic markers for VD. It has been reported that plasma miR-409-3p, miR-502-3p and miR-451a may be used to differentiate small-vessel VD patients from healthy controls (41). In addition, a number of miRNAs have been shown to be involved in synapses and may participate in the regulation of synaptic development, as well as functional regulation (42). A previous study reported that miR-134-5p is involved in the development and morphological changes of synapses (43). miR-134 is found in neurons and dendrites of primary cultured neurons, where it regulates the development of the dendritic spine by targeting LimK1 mRNA, which can be inhibited by BDNF (18). Furthermore, the overexpression of miR-134 leads to a markedly decreased the size of neuronal dendritic spines (33). miR-146, miR-125 and miR-485 were previously found to be involved in the regulation of synaptic plasticity, including control of dendritic spine number and 
A
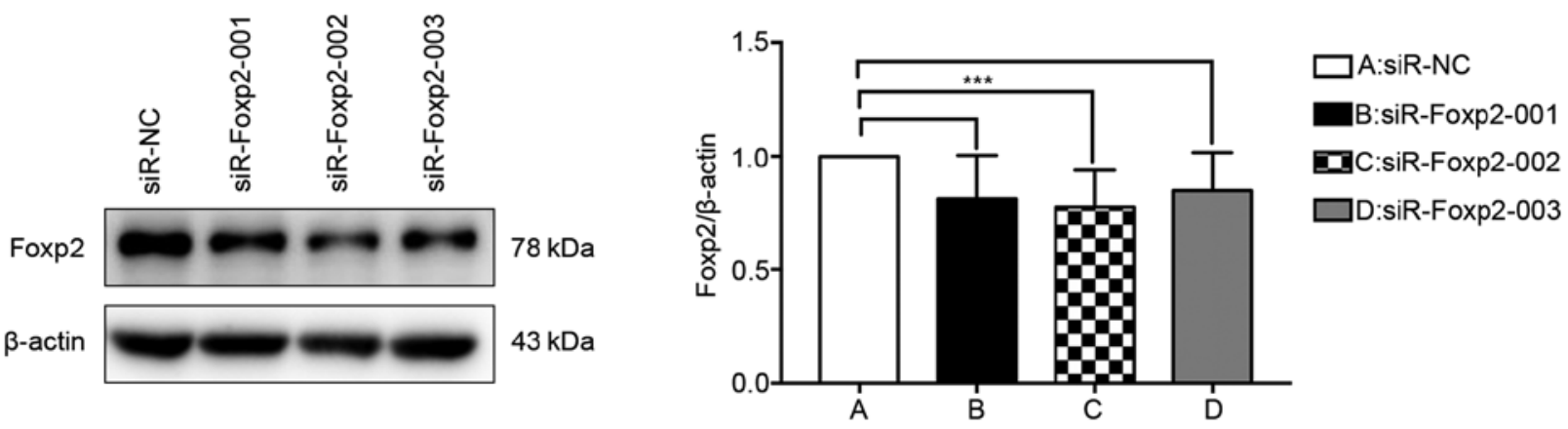

B
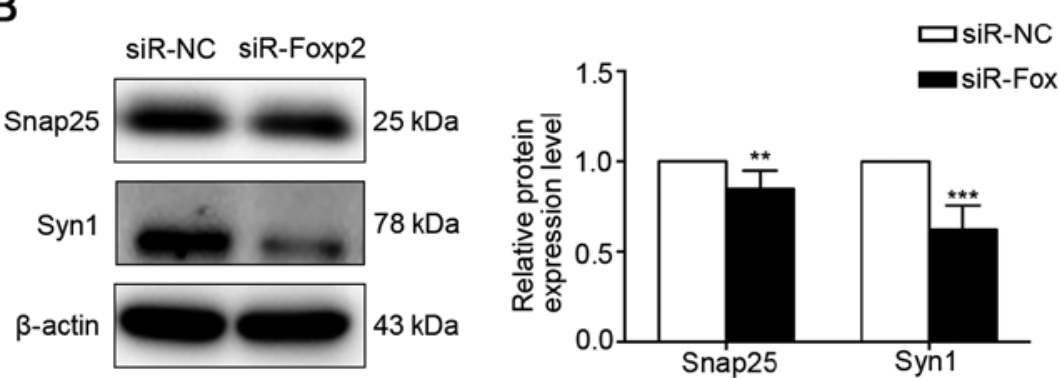

C

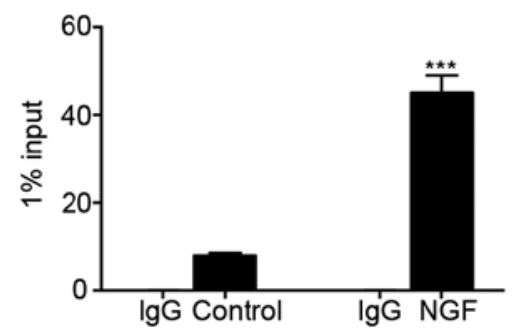

Figure 6. miR-134-5p indirectly regulates synaptic-associated proteins by regulating Foxp2, and Syn1 is a potential downstream target of Foxp2. (A) siRNA-Foxp2-002 was the most efficient sequence for the silencing of Foxp2 expression as determined using western blotting. ${ }^{* * *} \mathrm{P}<0.001 \mathrm{vs}$. the siR-NC group (one-way ANOVA). (B) Western blot analysis revealed that the expression of Syn1 and Snap25 was decreased following silencing of Foxp2. ${ }^{* *} \mathrm{P}<0.01$, ${ }_{* * * *} \mathrm{P}<0.001$ vs. the siR-NC group (Student's t-test). (C) Chromatin immunoprecipitation analysis demonstrated that Foxp2 binds to the promoter sequence of Syn1. ${ }^{* * * *} \mathrm{P}<0.001$ vs. the control group (one-way ANOVA). Data are presented as the mean \pm standard deviation (n=3/group). siRNA/siR, short interfering RNA; NC, negative control; Foxp2, forkhead box P2; Snap25, synaptosome-associated protein 25; Syn1, synapsin I; NGF, nerve growth factor.

synapse formation $(29,44-46)$. However, there is currently no definitive and effective method for preventing the progression of cognitive dysfunction.

The key finding of the present study was that miR-134-5p is involved in cognitive dysfunction in VD rats. RT-qPCR analysis revealed that miR-134-5p expression was increased in the cortex of VD rats; however, no significant change in hippocampal tissue was observed. Therefore, it was inferred that the cortex is one of the initial lesion sites in the early stage of VD induced by 4 weeks of ischemia. H\&E and TUNEL staining in the present study indicated that pathological damage is more prominent in the cortex of early VD rats compared with that in the hippocampus after chronic cerebral hypoperfusion for 4 weeks. Inhibition of miR-134-5p expression ameliorated the cerebral hypoperfusion-induced neuronal damage. This observation was consistent with the results of the MWM test. Specific deficits were found in animals with damage to the hippocampus, striatum, basal forebrain, cerebellum and several cortical areas, which may affect MWM performance in various ways (47).

Another important finding of the present study was that miR-134-5p contributes to the loss of synaptic proteins in VD rats. Western blotting and immunohistochemical staining suggested that the inhibition of miR-134-5p increased the loss of synaptic proteins. miR-134-5p was also demonstrated to regulate the expression of synaptic proteins in vivo and in vitro. A number of previous studies have reported that synaptic protein loss is associated with cognitive impairment in VD rats $(8,48)$. The present study revealed that miR-134-5p promotes cognitive impairment through the loss of synaptic proteins. According to the results of the histopathological analysis, the miR-134-5p antagomir significantly improved pathological tissue injury in the brains of VD rats.

An important mechanism of action of miR-134-5p was revealed in the present study. miR-134-5p was found to regulate the loss of synaptic proteins by targeting the mRNA of Foxp2. Foxp2 was identified as a target of miR-134-5p using bioinformatics target prediction tools. As a transcription factor, Foxp2 regulates the expression of hundreds of downstream target genes, many of which are involved in regulating neural development and function in different ways (49). A number of these target genes play important roles in synaptic plasticity, including axon growth, axon guidance and neurotransmission $(23,50)$. Foxp2 is enriched in the cerebral cortex, striatum and cerebellum of the rodent brain, which are areas participating in motor control $(51,52)$. In Foxp2 mutant mice, in addition to vocal cord defects, changes in synaptic plasticity and impaired motor learning ability have been observed (53).

To verify the effect of Foxp2 on synaptic proteins, siRNA experiments were conducted to silence Foxp2 in the present study. These experiments indicated that Foxp2 inhibited the expression of Syn1 and Snap25. The data confirmed that miR-134-5p promoted cognitive impairment in VD rats by inhibiting Foxp2 directly. Syn1 also acts as a downstream target gene of Foxp2 and regulates the development and alterations of synapses (54). ChIP-qPCR analysis suggested that Syn1 is a downstream target gene of Foxp2. Syn1 plays multiple roles in synaptic transmission and plasticity by differentially affecting important steps of synaptic vesicle trafficking in excitatory and inhibitory synapses (55). The results of the present study indicated that Foxp 2 directly binds to the Syn 1 promoter sequence to regulate synaptic damage. The Foxp2/Syn1 pathway may 
also represent a novel target for the functional study of the effect of Foxp2 on synaptic plasticity.

In conclusion, the results of the present study have several important clinical implications for VD. First, miR-134-5p may be a potential biomarker for the early diagnosis of VD. It was demonstrated that miR-134-5p aggravates cognitive impairment in VD rats by damaging cortical neurons and reducing synaptic proteins. Second, the data suggested that miR-134-5p promotes cognitive impairment in early VD rats by inhibiting Foxp2. Therefore, the miR-134-5p/Foxp2/Syn1 pathway may represent a novel target for early VD synaptic damage and molecular-based therapeutic strategies.

\section{Acknowledgements}

Not applicable.

\section{Funding}

The present study was supported by the National Natural Science Foundation of China (grant no. 81673770) and the Natural Science Foundation of Guangdong Province (grant no. 2017A030312009).

\section{Availability of data and materials}

The datasets generated and analyzed during the present study are available from the corresponding author on reasonable request.

\section{Authors' contributions}

XL performed the experiments and wrote the manuscript; RZ and ZW performed experiments, including the animal-associated experiments and molecular biology; WS and ZR provided experimental technical guidance; SZ and JZ provided technical assistance; DC conceived and designed the experiments, and secured the project funds. All authors have read and approved the final version of this manuscript for publication.

\section{Ethics approval and consent to participate}

All animal experiments were approved by the Care and Use of Experimental Animals Committee of Guangzhou University of Chinese Medicine and were performed according to the National Institute of Health Guide for the Care and Use of Laboratory Animals.

\section{Patient consent for publication}

Not applicable.

\section{Competing interests}

All authors declare that they have no competing interests.

\section{References}

1. Guo LL, Wang DS, Xu YY and Cui KG: Effects of IL-1 $\beta$ on hippocampus cell apoptosis and learning ability of vascular dementia rats. Eur Rev Med Pharmacol Sci 22: 6042-6048, 2018.
2. Yang JW, Wang XR, Zhang M, Xiao LY, Zhu W, Ji CS and Liu CZ: Acupuncture as a multifunctional neuroprotective therapy ameliorates cognitive impairment in a rat model of vascular dementia: A quantitative iTRAQ proteomics study. CNS Neurosci Ther 24: 1264-1274, 2018.

3. Yang HY, Liu Y, Xie JC, Liu NN and Tian X: Effects of repetitive transcranial magnetic stimulation on synaptic plasticity and apoptosis in vascular dementia rats. Behav Brain Res 281: 149-155, 2015.

4. Farkas E and Luiten PG: Cerebral microvascular pathology in aging and Alzheimer's disease. Prog Neurobiol 64: 575-611, 2001.

5. Damodaran T, Müller CP and Hassan Z: Chronic cerebral hypoperfusion-induced memory impairment and hippocampal long-term potentiation deficits are improved by cholinergic stimulation in rats. Pharmacol Rep 71: 443-448, 2019.

6. Waldmeier PC: Prospects for antiapoptotic drug therapy of neurodegenerative diseases. Prog Neuropsychopharmacol Biol Psychiatry 27: 303-321, 2003.

7. Jinglong T, Weijuan G, Jun L, Tao Q, Hongbo Z and Shasha L: The molecular and electrophysiological mechanism of buyanghuanwu decoction in learning and memory ability of vascular dementia rats. Brain Res Bull 99: 13-18, 2013.

8. Scheff SW, Neltner JH and Nelson PT: Is synaptic loss a unique hallmark of Alzheimer's disease? Biochem Pharmacol 88: 517-528, 2014.

9. Kalaria RN: The pathology and pathophysiology of vascular dementia. Neuropharmacology 134: 226-239, 2018.

10. Ren Z, Yu J, Wu Z, Si W, Li X, Liu Y, Zhou J, Deng R and Chen D: MicroRNA-210-5p contributes to cognitive impairment in early vascular dementia rat model through targeting Snap25. Front Mol Neurosci 11: 388, 2018

11. Terry RD, Masliah E, Salmon DP, Butters N, DeTeresa R, Hill R, Hansen LA and Katzman R: Physical basis of cognitive alterations in Alzheimer's disease: Synapse loss is the major correlate of cognitive impairment. Ann Neurol 30: 572-580, 1991.

12. Blennow K, Bogdanovic N, Alafuzoff I, Ekman R and Davidsson P: Synaptic pathology in Alzheimer's disease: Relation to severity of dementia, but not to senile plaques, neurofibrillary tangles, or the ApoE4 allele. J Neural Transm (Vienna) 103: 603-618, 1996.

13. Guo Y, Zhao Y, Nan Y, Wang X, Chen Y and Wang S: (-)-Epigallocatechin-3-gallate ameliorates memory impairment and rescues the abnormal synaptic protein levels in the frontal cortex and hippocampus in a mouse model of Alzheimer's disease. Neuroreport 28: 590-597, 2017.

14. Guarnieri FC, Pozzi D, Raimondi A, Fesce R, Valente MM, Delvecchio VS, Van Esch H, Matteoli M, Benfenati F, D'Adamo P and Valtorta F: A novel SYN1 missense mutation in non-syndromic X-linked intellectual disability affects synaptic vesicle life cycle, clustering and mobility. Hum Mol Genet 26: 4699-4714, 2017.

15. Batista AFR, Martinez JC and Hengst U: Intra-axonal synthesis of SNAP25 is required for the formation of presynaptic terminals. Cell Rep 20: 3085-3098, 2017.

16. Fassio A, Patry L, Congia S, Onofri F, Piton A, Gauthier J, Pozzi D, Messa M, Defranchi E, Fadda M, et al: SYN1 loss-of-function mutations in autism and partial epilepsy cause impaired synaptic function. Hum Mol Genet 20: 2297-2307, 2011.

17. Shupliakov O, Haucke V and Pechstein A: How synapsin I may cluster synaptic vesicles. Semin Cell Dev Biol 22: 393-399, 2011.

18. Sinclair LI, Tayler HM and Love S: Synaptic protein levels altered in vascular dementia. Neuropathol Appl Neurobiol 41: 533-543, 2015.

19. Gallart-Palau X, Serra A, Qian J, Chen CP, Kalaria RN and Sze SK: Temporal lobe proteins implicated in synaptic failure exhibit differential expression and deamidation in vascular dementia. Neurochem Int 80: 87-98, 2015.

20. Rodenas-Cuadrado PM, Mengede J, Baas L, Devanna P, Schmid TA, Yartsev M, Firzlaff U and Vernes SC: Mapping the distribution of language related genes FoxP1, FoxP2, and CntnaP2 in the brains of vocal learning bat species. J Comp Neurol 526: 1235-1266, 2018.

21. Chiu YC, Li MY, Liu YH, Ding JY, Yu JY and Wang TW: Foxp2 regulates neuronal differentiation and neuronal subtype specification. Dev Neurobiol 74: 723-738, 2014.

22. Vernes SC, Spiteri E, Nicod J, Groszer M, Taylor JM, Davies KE, Geschwind DH and Fisher SE: High-throughput analysis of promoter occupancy reveals direct neural targets of FOXP2, a gene mutated in speech and language disorders. Am J Hum Genet 81: 1232-1250, 2007. 
23. Konopka G, Bomar JM, Winden K, Coppola G, Jonsson ZO, Gao F, Peng S, Preuss TM, Wohlschlegel JA and Geschwind DH: Human-specific transcriptional regulation of CNS development genes by FOXP2. Nature 462: 213-217, 2009.

24. Spiteri E, Konopka G, Coppola G, Bomar J, Oldham M, Ou J, Vernes SC, Fisher SE, Ren B and Geschwind DH: Identification of the transcriptional targets of FOXP2, a gene linked to speech and language, in developing human brain. Am J Hum Genet 81: 1144-1157, 2007.

25. Vernes SC, Oliver PL, Spiteri E, Lockstone HE, Puliyadi R, Taylor JM, Ho J, Mombereau C, Brewer A, Lowy E, et al: Foxp2 regulates gene networks implicated in neurite outgrowth in the developing brain. PLoS Genet 7: e1002145, 2011.

26. Fu L, Shi Z, Luo G, Tu W, Wang X, Fang Z and Li X: Multiple microRNAs regulate human FOXP 2 gene expression by targeting sequences in its $3^{\prime}$ untranslated region. Mol Brain 7: 71, 2014.

27. Higuchi Y, Soga T and Parhar IS: Potential roles of microRNAs in the regulation of monoamine oxidase a in the brain. Front Mol Neurosci 11: 339, 2018.

28. Wang D, Wang X, Liu X, Jiang L, Yang G, Shi X, Zhang C and Piao F: Inhibition of miR-219 alleviates arsenic-induced learning and memory impairments and synaptic damage through up-regulating CaMKII in the hippocampus. Neurochem Res 43 948-958, 2018.

29. Edbauer D, Neilson JR, Foster KA, Wang CF, Seeburg DP, Batterton MN, Tada T, Dolan BM, Sharp PA and Sheng M: Regulation of synaptic structure and function by FMRP-associated microRNAs miR-125b and miR-132. Neuron 65: 373-384, 2010.

30. Wayman GA, Davare M, Ando H, Fortin D, Varlamova O, Cheng HY, Marks D, Obrietan K, Soderling TR, Goodman RH and Impey S: An activity-regulated microRNA controls dendritic plasticity by down-regulating p250GAP. Proc Natl Acad Sci USA 105: 9093-9098, 2008.

31. McCann C, Holohan EE, Das S, Dervan A, Larkin A, Lee JA, Rodrigues V, Parker R and Ramaswami M: The Ataxin-2 protein is required for microRNA function and synapse-specific long-term olfactory habituation. Proc Natl Acad Sci USA 108: E655-E662, 2011.

32. Schratt GM, Tuebing F, Nigh EA, Kane CG, Sabatini ME, Kiebler M and Greenberg ME: A brain-specific microRNA regulates dendritic spine development. Nature 439: 283-289, 2006.

33. Bicker S, Lackinger M, Weiß K and Schratt G: MicroRNA-132, -134, and -138: A microRNA troika rules in neuronal dendrites. Cell Mol Life Sci 71: 3987-4005, 2014.

34. Yu H, Fan C, Yang L, Yu S, Song Q, Wang P and Mao X: Ginsenoside Rg1 prevents chronic stress-induced depression-like behaviors and neuronal structural plasticity in rats. Cell Physiol Biochem 48: 2470-2482, 2018.

35. Livak KJ and Schmittgen TD: Analysis of relative gene expression data using real-time quantitative PCR and the 2(-Delta Delta C(T)) method. Methods 25: 402-408, 2001.

36. Liu C, Guo Y, Zhao F, Qin H, Lu H, Fang L, Wang J and Min W: Potential mechanisms mediating the protective effects of a peptide from walnut (Juglans mandshurica Maxim.) against hydrogen peroxide induced neurotoxicity in PC12 cells. Food Funct 10: 3491-3501, 2019.

37. Niu XL, Jiang X, Xu GD, Zheng GM, Tang ZP, Yin N, Li XQ, Yang YY and Lv PY: DL-3-n-butylphthalide alleviates vascular cognitive impairment by regulating endoplasmic reticulum stress and the Shh/Ptch1 signaling-pathway in rats. J Cell Physiol 234: 12604-12614, 2019.

38. Komatani A, Yamaguchi K, Sugai Y, Takanashi T, Kera M, Shinohara M and Kawakatsu S: Assessment of demented patients by dynamic SPECT of inhaled xenon-133. J Nucl Med 29: $1621-1626,1988$

39. Ohnishi T, Hoshi H, Nagamachi S, Jinnouchi S, Flores LG II, Futami S and Watanabe K: High-resolution SPECT to assess hippocampal perfusion in neuropsychiatric diseases. J Nucl Med 36: 1163-1169, 1995.
40. Tsuchiya M, Sako K, Yura S and Yonemasu Y: Local cerebral glucose utilisation following acute and chronic bilateral carotid artery ligation in Wistar rats: Relation to changes in local cerebral blood flow. Exp Brain Res 95: 1-7, 1993.

41. Prabhakar P, Chandra SR and Christopher R: Circulating microRNAs as potential biomarkers for the identification of vascular dementia due to cerebral small vessel disease. Age Ageing 46: 861-864, 2017.

42. Baek D, Villén J, Shin C, Camargo FD, Gygi SP and Bartel DP: The impact of microRNAs on protein output. Nature 455: 64-71, 2008.

43. McGowan H, Mirabella VR, Hamod A, Karakhanyan A, Mlynaryk N, Moore JC, Tischfield JA, Hart RP and Pang ZP: Hsa-let-7c miRNA regulates synaptic and neuronal function in human neurons. Front Synaptic Neurosci 10: 19, 2018.

44. Prada I, Gabrielli M, Turola E, Iorio A, D'Arrigo G, Parolisi R, De Luca M, Pacifici M, Bastoni M, Lombardi M, et al: Glia-to-neuron transfer of miRNAs via extracellular vesicles: A new mechanism underlying inflammation-induced synaptic alterations. Acta Neuropathol 135: 529-550, 2018.

45. Wang X,Liu D,Huang HZ, WangZH,HouTY, Yang X,PangP, WeiN, Zhou YF, Dupras MJ, et al: A novel microRNA-124/PTPN1 signal pathway mediates synaptic and memory deficits in Alzheimer's disease. Biol Psychiatry 83: 395-405, 2018.

46. Cohen JE, Lee PR, Chen S, Li W and Fields RD: MicroRNA regulation of homeostatic synaptic plasticity. Proc Natl Acad Sci USA 108: 11650-11655, 2011

47. D'Hooge R and De Deyn PP: Applications of the Morris water maze in the study of learning and memory. Brain Res Brain Res Rev 36: 60-90, 2001.

48. Zhu Y, Zhang Q, Zhang W, Li N, Dai Y, Tu J, Yang F, Brann DW and Wang R: Protective effect of $17 \beta$-estradiol upon hippocampal spine density and cognitive function in an animal model of vascular dementia. Sci Rep 7: 42660, 2017.

49. Estruch SB, Graham SA, Quevedo M, Vino A, Dekkers DHW Deriziotis P, Sollis E, Demmers J, Poot RA and Fisher SE: Proteomic analysis of FOXP proteins reveals interactions between cortical transcription factors associated with neurodevelopmental disorders. Hum Mol Genet 27: 1212-1227, 2018.

50. Hachigian LJ, Carmona V, Fenster RJ, Kulicke R, Heilbut A, Sittler A, Pereira de Almeida L, Mesirov JP, Gao F, Kolaczyk ED and Heiman M: Control of huntington's disease-associated phenotypes by the striatum-enriched transcription factor Foxp2. Cell Rep 21: 2688-2695, 2017.

51. Ferland RJ, Cherry TJ, Preware PO, Morrisey EE and Walsh CA Characterization of Foxp2 and Foxp1 mRNA and protein in the developing and mature brain. J Comp Neurol 460: 266-279, 2003.

52. Hisaoka T, Nakamura Y, Senba E and Morikawa Y: The forkhead transcription factors, Foxp1 and Foxp2, identify different subpopulations of projection neurons in the mouse cerebral cortex. Neuroscience 166: 551-563, 2010.

53. Groszer M, Keays DA, Deacon RM, de Bono JP, PrasadMulcare S, Gaub S, Baum MG, French CA, Nicod J, Coventry JA, et al: Impaired synaptic plasticity and motor learning in mice with a point mutation implicated in human speech deficits. Curr Biol 18: 354-362, 2008.

54. Kang HJ, Voleti B, Hajszan T, Rajkowska G, Stockmeier CA, Licznerski P, Lepack A, Majik MS, Jeong LS, Banasr M, et al: Decreased expression of synapse-related genes and loss of synapses in major depressive disorder. Nat Med 18: 1413-1417, 2012.

55. Park HJ, Kim SK, Kang WS, Chung JH and Kim JW: Increased activation of synapsin 1 and mitogen-activated protein kinases/extracellular signal-regulated kinase in the amygdala of maternal separation rats. CNS Neurosci Ther 20: 172-181, 2014.

This work is licensed under a Creative Commons

Attribution-NonCommercial-NoDerivatives 4.0

International (CC BY-NC-ND 4.0) License. 